\title{
Reverse Transport of Cholesterol Is the Reason for Resistance to Development of Atherosclerosis in Prague Hereditary Hypercholesterolemic (PHHC) Rat
}

\author{
M. SCHMIEDTOVA ${ }^{1}$, M. HECZKOVA ${ }^{1}$, J. KOVAR $^{1}$, I. KRALOVA LESNA ${ }^{1}$, \\ R. POLEDNE ${ }^{1}$
}

${ }^{1}$ Institute of Clinical and Experimental Medicine, Prague, Czech Republic

Received October 3, 2013

Accepted April 16, 2014

On-line June 5, 2014

\section{Summary}

The Prague Hereditary Hypercholesterolemic (PHHC) rat is a model of hypercholesterolemia. In previous experiments, it was found to be completely resistant to the development of atherosclerosis. It was assumed that the reverse transport of cholesterol (RCT) might be the reason for this resistance. In this study, RCT was measured in vivo by cholesterol efflux from macrophages to plasma, using previously established methods for RCT in mice (Rader 2003), optimized for measurements in rats. Primary cell culture of macrophages was labeled with ${ }^{14} \mathrm{C}$ cholesterol and then injected intraperitoneally into rats. Plasma and feces were collected at 24 and $48 \mathrm{~h}$. The plasma ${ }^{14} \mathrm{C}$ cholesterol levels at both 24 and $48 \mathrm{~h}$ were significantly higher in male $\mathrm{PHHC}$ rats compared to control Wistar rats. The $\mathrm{PHHC}$ rats excreted less ${ }^{14} \mathrm{C}$-cholesterol in feces in 24 and $48 \mathrm{~h}$ compared to Wistar rats. The largest pool of ${ }^{14} \mathrm{C}$-cholesterol was found in the adipose tissue of $\mathrm{PHHC}$ rats and in contrast lower levels of ${ }^{14} \mathrm{C}$ cholesterol were measured in the liver and muscle tissues of PHHC rats compared with Wistar rats. Increasing release of ${ }^{14} \mathrm{C}$ cholesterol efflux from macrophages demonstrates accelerated RTC and leads to prevention of atherogenesis in PHHC rats.

\section{Key words}

Macrophages • Atherosclerosis $\bullet$ Cholesterol

\section{Corresponding author}

R. Poledne, Institute of Clinical and Experimental Medicine, Videnska 1958/9, 14021 Prague 4, Czech Republic. E-mail: rupo@medicon.cz

\section{Introduction}

LDL cholesterol and macrophages play important roles in the initiation and progression of atherosclerotic lesions (Fielding and Fielding 1995). Accumulation of cholesterol within arterial walls increases the risk of atherosclerosis and cardiovascular diseases. Reverse cholesterol transport is the opposing movement of cholesterol from the walls of arteries through the plasma compartment to the liver for excretion in bile present in feces. RCT represents a protective mechanism against atherosclerosis development (Rader 2003, Zhao et al. 2007), but not every cholesterol efflux from tissues is a part of the classic RCT model. In reality, a decreased cholesterol efflux from the cells in the arterial wall has the potential to transform invading monocytes into foam cells. Primary macrophages are the most convenient model for analyzing RCT and studying atherosclerosis thanks to identical homology in animal models (Cuchel and Rader 2006).

The most frequently used animal model for RCT measurement is mouse and, in addition, the apoE $\mathrm{KO}$ mouse is the most frequently used model of atherosclerosis among rodents (Annema and Tietge 2012, Plump et al. 1992, Zhang et al. 1992). In contrast, rats have not been used as a model in atherosclerosis research at all. The Prague hereditary hypercholesterolemic (PHHC) rat was obtained by selection and brother-sister inbreeding from a Wistar strain in our laboratory (Poledne 1986). PHHC rat is a model of hypercholesterolemia induced by dietary cholesterol 
without any addition of cholic acid or antithyroid drugs. Hypercholesterolemia is induced in other strains of rats only when cholic acid added to dietary cholesterol blocks the basic metabolic pathway of cholesterol elimination. Cholic acid is generally used in a quantity to produce hypercholesterolemia, but this quantity is hepatotoxic for rats (Delzenne et al. 1992). These are limits for usage of rat models in atherosclerosis research. PHHC rats have modestly increased cholesterolemia when fed with a standard chow diet and on a $2 \%$ cholesterol diet (without any addition of cholic acid) with hypercholesterolemia exceeding $10 \mathrm{mmol} / \mathrm{l}$ in males and $15 \mathrm{mmol} / \mathrm{l}$ in females, respectively. The feeding of rats with the cholesterol diet results in development of fatty liver. After feeding PHHC rats with a cholesterol diet, the lipoprotein profile is substantially changed and it is more similar to hyperlipoproteinemia in humans. The most pronounced changes were found in very low density lipoproteins (VLDL) and intermediate density lipoproteins (IDL). Most cholesterol was carried out in VLDL and IDL particles (Hubacek et al. 2008). Cholesterol concentration in low density lipoproteins (LDL) was also increased (Kovar et al. 2009). Thus, the PHHC rat seems to be a very convenient model to study the mechanism involved in the regulation of cholesterolemia and atherosclerosis and it is closer to humans than other rat models of hypercholesterolemia as well.

In our previous experiment, rats (males) were kept for 6 months on a $2 \%$ cholesterol diet with cholesterolemia reaching $18 \pm 10 \mathrm{mmol} / \mathrm{l}$. Although PHHC rats develop cholesterol concentration similar to familiar hypercholesterolemia when fed with a cholesterol diet, no atherosclerosis was observed. No macroscopic lesions were found in the aorta immediately after killing these animals and no differences in histology of ascending thoracic aorta between PHHC rats on cholesterol diet and those of the same age kept on chow were found (Kovar et al. 2009).

One of the reasons for the resistance to the development of atherosclerosis in the PHHC rat could certainly be the accelerated reverse transport of cholesterol. In this study, RCT in vivo was evaluated by measuring the cholesterol efflux from macrophages to plasma using previously established methods for RCT in mice (Rader 2003). Using this method, we assumed that the outflow of cholesterol from macrophages into the circulation and body compartments is low or close to zero before injecting ${ }^{14}$ C-cholesterol. This method was optimized for measurements in rats using homologous macrophages.

\section{Materials and Methods}

Animals

Prague hereditary hypercholesterolemic (PHHC) rats (male), which were selected as an inbred strain from Wistar rats in our laboratory at The Institute of Clinical and Experimental Medicine (IKEM), were used for the studies. Male Wistar rats as a control group for the experiments were obtained from Anlab s.r.o. Rats were 10-12 weeks old, housed in cages ( 1 animal per each cage) with a 12-h light/dark cycle. Animals were kept on standard chow diet before experiment and had free access to food and water.

\section{Optimization of macrophages labeling}

Primary macrophages were obtained from

8 male PHHC rats. Donor rats were injected intraperitoneally with $2 \mathrm{ml}$ of $10 \%$ thioglycollate (Maggini et al. 2010). After 3 days, peritoneal macrophages were harvested by lavage using PBS + EDTA. These primary macrophages were then plated in RPMI 1640 medium supplemented with $10 \%$ fetal bovine serum and $0.5 \%$ gentamicin (Naik et al. 2006). Non-adherent cells were removed by washing twice with PBS and macrophages were labeled with $5 \mu \mathrm{Ci} / \mathrm{ml}{ }^{14} \mathrm{C}$ cholesterol dissolved in ethanol without any addition of acetylated LDL. At the end of labeling, these cells were washed twice with PBS again and equilibrated in medium, spun down and resuspended in $1 \mathrm{ml}$ of RPMI medium. Radioactivity of ${ }^{14} \mathrm{C}$-cholesterol-labeled macrophages was measured after 1, 4, 24 and $48 \mathrm{~h}$ incubation (Fig. 1) in Liquid Scintillation Counted (LSC).

\section{In vivo reverse cholesterol transport study}

Eight male PHHC rats and eight male Wistar rats were intraperitoneally injected with ${ }^{14} \mathrm{C}$-cholesterollabeled cells $\left(8 \times 10^{5}\right.$ cells containing $4 \times 10^{5} \mathrm{dpm}$ in $0.5 \mathrm{ml}$ of medium) prepared as described above. Rats were kept individually in cages. Blood was collected at $24 \mathrm{~h}$ (tail vein) and 48 h (vena cava) and plasma was directly used for LSC. Feces were collected continuously within intervals of $0-24 \mathrm{~h}$ and 24-48 h, respectively. Liver, perirenal adipose tissue and muscles of femur tissues were removed and frozen in liquid nitrogen until lipid extraction was performed.

\section{Extraction of fecal cholesterol and its metabolites}

Fecal samples were dried, weighed, and thoroughly ground. Samples were soaked in Millipore 
water overnight at $4{ }^{\circ} \mathrm{C}$. The next day ethanol was added and the samples were homogenised. An aliquot of each extraction mixture was measured in LSC (Iverson et al. 2001).

\section{Tissues lipid extraction}

Tissue lipids were extracted according to the procedure of Blight and Dyer (Zhang et al. 2003, Matyash et al. 2008). Briefly, tissue was homogenised in water, and then lipids were extracted with a mixture of chloroform, methanol and water in ratio 1:2:0.8. The solution was rehomogenized with chloroform, followed by distilled water. The ratio of chloroform, methanol and water then was 2:2:1.8. Samples were centrifuged which led to the creation of a two-phase system. The lower (chloroform) lipid phase was collected, solvents were evaporated under nitrogen and lipids were resuspended in toluene and measured in LSC.

\section{Results}

\section{Optimization of macrophages labeling}

The first objective of this study was to obtain highly labeled in vitro primary macrophages with good viability. For carrying out the experiment, it was necessary to pre-determine the optimal time of loading of macrophages with ${ }^{14} \mathrm{C}$-cholesterol to get a high level of radioactivity without any supplementation of acLDL. The high level of radioactivity in macrophages is needed for the subsequent detection of ${ }^{14} \mathrm{C}$-cholesterol in the body of the experimental animal after in vivo application. Total number of cells collected from peritoneal cavities of all rats was $9.2 \pm 1.8 \times 10^{6}(\mathrm{n}=8)$. Primary cell culture of macrophages from rats was incubated with ${ }^{14} \mathrm{C}$-cholesterol for 1, 2, 24 and $48 \mathrm{~h}$. As shown in Figure 1, the radioactivity of ${ }^{14} \mathrm{C}$-cholesterol labeled primary cell culture was increasing through time with the maximum at $48 \mathrm{~h}$, when the radioactivity was $6.7 \times 10^{5} \mathrm{dpm}$. On the other hand, cell viability was decreasing during the whole experiment, but more than $85 \%$ of macrophages still fulfilled the criteria of viability after $48 \mathrm{~h}$ of incubation. Moreover, $48 \mathrm{~h}$ of incubation led to the sufficient incorporation of ${ }^{14} \mathrm{C}$ cholesterol into the cells. We therefore concluded that the labeling of macrophages in vitro as documented by Zhao et al. (2007) in mice is applicable also in rats. The $48 \mathrm{~h}$ labeling in vitro was consequently used in all our experiments.

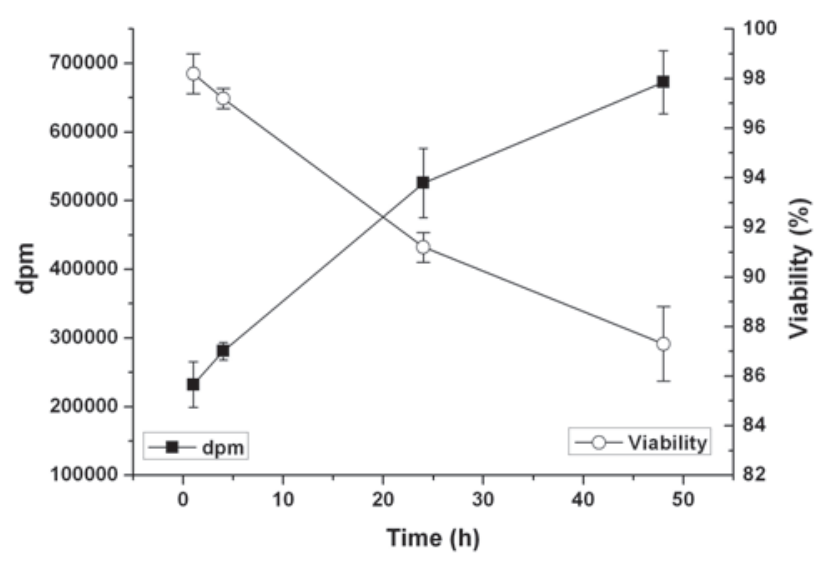

Fig. 1. Optimizing time of labeling.

In vivo reverse cholesterol transport study

The second objective of our study was to verify feasibility of measuring reverse cholesterol transport by using pre-labeled macrophages and measure reverse cholesterol transport in rats.

Total number of the cells collected from peritoneal cavities of the donor PHHC rat after their stimulation by thioglycolate was $8.1 \pm 2.1 \times 10^{6}(\mathrm{n}=8)$ and total number of cells collected from donor Wistar rat peritoneal cavities was $28.1 \pm 4.5 \times 10^{6} \quad(\mathrm{n}=8)$. After labeling with ${ }^{14} \mathrm{C}$-cholesterol, $8 \times 10^{5}$ cells contained $4 \times 10^{5} \mathrm{dpm}$ in $0.5 \mathrm{ml}$ of medium and viability of the cells was $86 \pm 5.1 \%$.

8 male PHHC rats and 8 male Wistar rats were injected by of homologous primary cell culture of macrophages containing ${ }^{14} \mathrm{C}$-cholesterol. The amount of radioactivity applied to one animal was $8 \times 10^{5} \mathrm{dpm}$ in $1 \mathrm{ml}$ of medium.

As demonstrated in Figure 2, 5.79 $\pm 0.65 \%$ of total applied radioactivity was found in the total plasma volume of the PHHC rats whereas a significantly lower ratio $4.56 \pm 0.18 \%$ was found in the total plasma volume of the control rats. The similar ratio was found two days after application of labeled macrophages. There was $5.32 \pm 0.32 \%$ radioactivity applied in the PHHC rats compared to $3.84 \pm 0.22 \%$ in the controls. The slight decrease of total radioactivity measured after $48 \mathrm{~h}$ was observed compared to the values after $24 \mathrm{~h}$, while the relative difference between PHHC rats and controls was increased (ratio 1.27:1.39). These data simply documented that the outflow of labeled cholesterol from the pre-labeled macrophages was significantly higher in the PHHC rats compared to controls which demonstrated accelerated RTC analyses in vivo PHHC rats. 


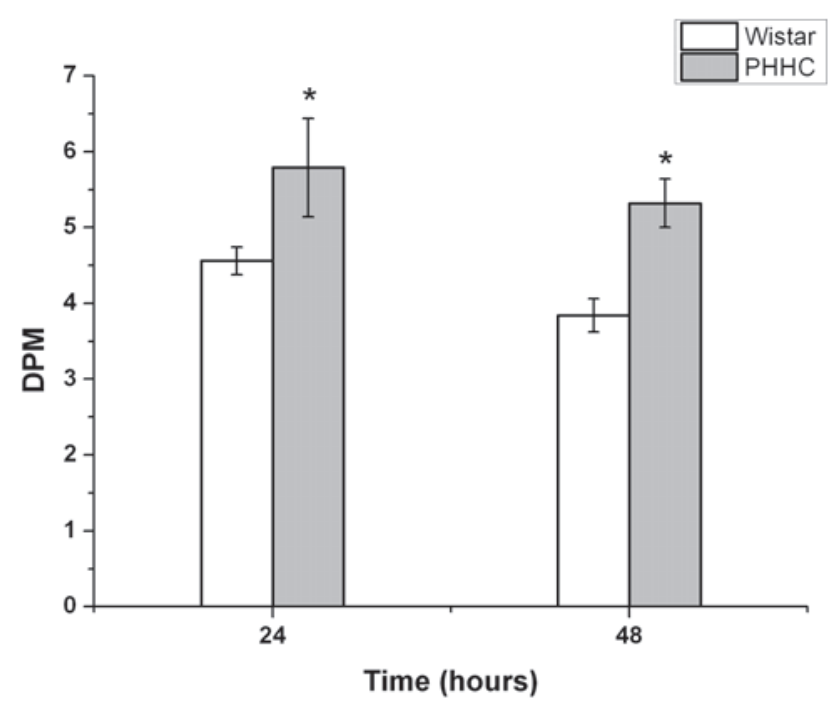

Fig. 2. The plasma ${ }^{14} \mathrm{C}$-cholesterol levels at 24 and $48 \mathrm{~h}$. Values are mean \pm S.E.M. * indicates difference from corresponding value in Wistar rats; $\mathrm{P}<0.005$, Student's $\mathrm{t}$ test.

Feces were collected during first $24 \mathrm{~h}$ and at the end of experiment after $48 \mathrm{~h}$. The relative differences between PHHC rats and controls were similar both the first and second day. Radioactivity in the second day was higher compared to the first $24 \mathrm{~h}$. Surprisingly, the total radioactivity in the feces of the PHHC rats was significantly lower compared to controls despite of the accelerated reverse cholesterol transport from injected pre-labeled macrophages.

Table 1. The ${ }^{14} \mathrm{C}$-cholesterol levels in tissue - liver, perirenal adipose tissue and quadriceps femoris muscle after $48 \mathrm{~h}$.

\begin{tabular}{lcc}
\hline Tissues & Wistar & PHHC \\
\hline $\begin{array}{l}\text { Liver }(\mathrm{dpm} / \mathrm{g}) \\
\text { Perirenal } \\
\text { adipose tissue } \\
\text { (dpm/g) }\end{array}$ & $6110.03 \pm 637.72$ & $4243.34 \pm 502.48^{*}$ \\
Muscle $(\mathrm{dpm} / \mathrm{g})$ & $2599.84 \pm 817.36$ & $1768.36 \pm 489.85$ \\
\hline
\end{tabular}

Values are mean \pm S.E.M. * indicates difference from corresponding value in Wistar rats; $P<0.005$, Student's $t$ test.

After decapitation of the animals, the liver, perirenal adipose tissues and skeletal muscles were extracted and radioactivity was determined. Radioactivity of the liver in PHHC rats was significantly lower compared to control group whereas the opposite ratio was found in the adipose tissues. The difference in radioactivity of perirenal adipose tissues was $7332 \pm 1106 \mathrm{dpm}$ per gram in PHHC rats compared to $4879 \pm 367 \mathrm{dpm}$ per gram in controls. Only the differences in the liver and adipose tissues were higher significantly $(\mathrm{P}<0.005)$. The radioactivity in the muscle was not found significantly different although the ratio in the control rats displayed an increased trend in Table 1.

\section{Discussion}

\section{Optimization of macrophages labeling}

From analysis of the time curve of radioactivity incorporated into peritoneal macrophages in the tissue culture experiment, it is evident that radioactivity increases almost linearly during $24 \mathrm{~h}$ and the rate of labeling slightly decreases during the following $24 \mathrm{~h}$ of incubation. The viability of the macrophages in the primary culture incubation decreased slightly at this time but still more than $85 \%$ of the incubation cells remained viable after $48 \mathrm{~h}$ and the differences in viability between 24 and $48 \mathrm{~h}$ were very small. Based on these data, we decided to pre-label macrophages by $48 \mathrm{~h}$ incubation to obtain higher radioactivity in the cells used for in vivo labeling of cholesterol pathways.

\section{In vivo reverse cholesterol transport study}

We decided to perform in vivo RTC analysis to find differences between two groups of rats of the same genetic origin. The difference between Wistar and PHHC rats lies in the individual RCT steps and also in the rate of RCT. Previous experiments showed no obvious difference in the lipoprotein profile between Wistar and PHHC rats kept on the standard chow diet. When fed with a control diet, both strains of Wistar and PHHC rats display a similar composition of lipoproteins and the majority of cholesterol molecules are located in HDL particles (Kovar et al. 2009). However, on high cholesterol diet, a substantial differences of lipoprotein concentrations between control Wistar and PHHC rats appear. The increased concentration of total cholesterol is 5-7 times higher in PHHC rats and this increase is most significant in intermediate density as well as low density lipoproteins.

In this experiment, only a standard chow diet was used to compare differences between PHHC and Wistar rats in cholesterol pathways. Reverse cholesterol transport is a complicated process including transfer of free cholesterol from cellular ABC receptors of extrahepatic cells (including macrophages) to HDL 
acceptors, its esterification and following exchange with apoprotein B containing lipoproteins (Fielding and Fielding 1995). We estimated the rate of all these pathways by release of total ${ }^{14} \mathrm{C}$-cholesterol radioactivity of prelabeled macrophages to the plasma pool. This very complex process of RTC has usually been measured by in vitro analysis in almost all published studies (Fielding and Fielding 1995) at present. In this methodological approach, the first part of cholesterol pathway in RCT is analyzed and the transfer from cellular membrane to lipoproteins acceptors is measured. In contrast to this situation, our in vivo approach is able to estimate this more complex process as a whole. The transfer of total radioactivity in the plasma pool is the sum of all steps from prelabeled macrophages to other lipoproteins in the plasma pool. Analyzing radioactivity of cholesterol in the plasma pool makes it possible to measure the entire pathways at once. On the other hand, the values of actual radioactivity measured in the plasma neglect the outflow of radioactivity from the plasma pool during the first and second day after the injection of labeled macrophages. This simplification is based on substantially different specific radioactivity of the pool of pre-labeled injected macrophages compared to diluted radioactivity of the total plasma pool. With this assumption, the data of measured RCT in vivo documented a higher rate of radioactivity appearing in plasma from pre-labeled macrophages. This transfer of radioactivity might be simply considered as a first order reaction. The higher radioactivity in plasma of $\mathrm{PHHC}$ rats demonstrated a faster release of cholesterol from the pre-labeled macrophages which represent accelerated RTC transport in vivo. During the experimental period, the label was distributed to all organs and the largest part of total labeled cholesterol was located in the liver, adipose tissues and muscle. Surprisingly, lower radioactivity was found in the liver of PHHC rats compared to controls whereas a higher proportion was found in the adipose tissues of PHHC rats. This study does not contain a detailed analysis of radioactive transport and its relative distribution into different organs. Our data obtained $48 \mathrm{~h}$ after injection of a label predicate that there probably exists a smaller pool in the liver of PHHC rat through which an accelerated rate of radioactivity from the prelabeled macrophages is transported to the depot pool of the adipose tissue. There were no differences in the radioactivity of the muscle tissues of both groups compared.

We decided to apply an in vivo approach for analyses of RCT in PHHC rats. This method and application was well documented by Zhao et al. (2007) in mice. Unfortunately, this result is not accompanied by higher radioactivity after $48 \mathrm{~h}$ in the feces of the PHHC rats. To follow the appearance of radioactivity in feces, a further experiment will have to last for a longer time. Also, the size of compartment in the adipose tissue of both compared strains need not be the same.

In conclusion, we were able to document that RCT determined in vivo was accelerated in PHHC rats compared to controls. According to our hypothesis, the acceleration of this pathway might be the reason for the resistance to atherosclerosis in $\mathrm{PHHC}$ rats in spite of the fact that this inbred strain is able to increase the concentration of atherogenic lipoproteins substantially. In our previous experiment, the long lasting increased concentration of apoB particles did not precede any atherosclerotic changes in the aorta.

\section{Conflict of Interest}

There is no conflict of interest.

\section{Acknowledgements}

The project was supported by MH CZ - DRO ("Institute for Clinical and Experimental Medicine - IKEM, IN 00023001”).

\section{References}

ANNEMA W, TIETGE UJ: Regulation of reverse cholesterol transport - a comprehensive appraisal of available animal studies. Nutr Metab (Lond) 9: 25, 2012.

CUCHEL M, RADER DJ: Macrophage reverse cholesterol transport: key to the regression of atherosclerosis? Circulation 113: 2548-2555, 2006.

DELZENNE NM, CALDERON PB, TAPER HS, ROBERFROID MB: Comparative hepatotoxicity of cholic acid, deoxycholic acid and lithocholic acid in the rat: in vivo and in vitro studies. Toxicol Lett 61: 291-304, 1992.

FIELDING CJ, FIELDING PE: Molecular physiology of reverse cholesterol transport. J Lipid Res 36: 322-331, 1995. 
HUBACEK JA, BOBKOVA D, BOHUSLAVOVA R, POLEDNE R: Differences in expression of cholesterol $7 \alpha-$ hydroxylase between PHHC and Wistar rats. Folia Biol (Praha) 54: 18-23, 2008.

IVERSON SJ, LANG SL, COOPER MH: Comparison of the Bligh and Dyer and Folch methods for total lipid determination in a broad range of marine tissue. Lipids 36: 1283-1287, 2001.

KOVAR J, TONAR Z, HECZKOVA M, POLEDNE R: Prague hereditary hypercholesterolemic (PHHC) rat - a model of polygenic hypercholesterolemia. Physiol Res 58: 95-99, 2009.

MAGGINI J, MIRKIN G, BOGNANNI I, HOLMBERG J, PIAZZÓN IM, NEPOMNASCHY I, COSTA H, CANONES C, RAIDEN S, VERMEULEN M, GEFFNER JR: Mouse bone marrow-derived mesenchymal stromal cells turn activated macrophages into a regulatory-like profile. PLoS One 5: e9252, 2010.

MATYASH V, LIEBISCH G, KURZCHALIA TV, SHEVCHENKO A, SCHWUDKE D: Lipid extraction by methyltert-butyl ether for high-throughput lipidomics. J Lipid Res 49: 1137-1146, 2008.

NAIK SU, WANG X, DA SILVA JS, JAYE M, MACPHEE CH, REILLY MP, BILLHEIMER JT, ROTHBLAT GH, RADER DJ: Pharmacological activation of liver $\mathrm{X}$ receptors promotes reverse cholesterol transport in vivo. Circulation 113: 90-97, 2006.

PLUMP AS, SMITH JD, HAYEK T, AALTO-SETALA K, WALSH A, VERSTUYFT JG, RUBIN EM, BRESLOW JL: Severe hypercholesterolemia and atherosclerosis in apolipoprotein E-deficient mice created by homologous recombination in ES cells. Cell 71: 343-353, 1992.

POLEDNE R: Effect of diet on cholesterol metabolism in Prague hereditary hypercholesterolemic rat. In: Nutritional Effects on Cholesterol Metabolism. AC BEYNEN (ed), Transmondial, Voorthuizen, 1986, pp 91-97.

RADER DJ: Regulation of reverse cholesterol transport and clinical implications. Am J Cardiol 92: 42J-49J, 2003.

ZHANG SH, REDDICK RL, PIEDRAHITA JA, MAEDA N: Spontaneous hypercholesterolemia and arterial lesions in mice lacking apolipoprotein E. Science 258: 468-471, 1992.

ZHANG Y, ZANOTTI I, REILLY MP, GLICK JM, ROTHBLAT GH, RADER DJ: Overexpression of apolipoprotein A-I promotes reverse transport of cholesterol from macrophages to feces in vivo. Circulation 108: 661-663, 2003.

ZHAO B, SONG J, CHOW WN, ST CLAIR RW, RUDEL LL, GHOSH S: Macrophage-specific transgenic expression of cholesteryl ester hydrolase significantly reduces atherosclerosis and lesion necrosis in Ldlr mice. J Clin Invest 117: 2983-2992, 2007. 